\title{
In vitro Anti-angiogenic Effects of Tea Saponin and Tea Aglucone on Human Umbilical Vein Endothelial Cells
}

\author{
Xiaohong Li ${ }^{1,2}$, Baozhan Huang ${ }^{1}$, Fan Fei ${ }^{1,2}$, Hai Niu ${ }^{1,3,}$, Wen Huang ${ }^{1, *}$ \\ ${ }^{1}$ Laboratory of Ethnopharmacology, Regenerative Medicine Research Center, Institute for Nanobiomedical Technology and Membrane \\ Biology, West China Hospital/West China Medical School, Sichuan University, Chengdu, P.R.China \\ ${ }^{2}$ Department of Biopharmaceutics, Key Laboratory of Drug Targeting and Drug Delivery Systems, Ministry of Education, West China \\ School of Pharmacy, Sichuan University, Chengdu, P.R. China \\ ${ }^{3}$ College of Mathematics, Sichuan University, Chengdu, P.R. China \\ *Corresponding author: niuhai@scu.edu.cn; huangwen@scu.edu.cn
}

Received February 05, 2015; Revised March 21, 2015; Accepted March 25, 2015

\begin{abstract}
Green tea is a popular beverage world-wide, especially in Asian countries. Its health benefits, derived from the Camellia sinensis leaves, have been studied over the years. However, few reports are available about the health effects of tea saponin (TS), an important component of Camellia sinensis leaves, and tea aglucone (TA). In this present study, we investigated the effect of TS and TA on the proliferation, migration, invasion and tube formation of human umbilical vein endothelial cells (HUVECs). TS inhibited the proliferation of HUVECs in a dose-dependent manner, with an IC50 of $7.5 \pm 0.6 \mu \mathrm{M}$. Inconsistent with the well-reported mechanisms of apoptotic induction and cell cycle arrest by steroidal saponins, effects of TS on apoptosis and cell cycle progression in HUVECs were not detected. The autophagic vacuoles in the TS-treated HUVECs, observed with transmission electron microscopy, suggested the involvement of autophagic induction in the growth inhibition of HUVECs. When treated with TS, cell migration, invasion and tube formation potency of HUVECs were markedly suppressed. TA also suppressed the proliferation of HUVECs, with an IC50 of $25.3 \pm 1.2 \mu \mathrm{M}$. In comparison with TS, TA inhibited the migration, invasion, and tube formation of HUVECs less effectively. Our data about the anti-angiogenic effects of TS and TA, presented for the first time, would provide a new insight into the health potential for green tea.
\end{abstract}

Keywords: tea saponin, tea aglucone, anti-angiogenic effects, human umbilical vein endothelial cells

Cite This Article: Xiaohong Li, Baozhan Huang, Fan Fei, Hai Niu, and Wen Huang, "In vitro Antiangiogenic Effects of Tea Saponin and Tea Aglucone on Human Umbilical Vein Endothelial Cells.” Journal of Food and Nutrition Research, vol. 3, no. 3 (2015): 206-212. doi: 10.12691/jfnr-3-3-13.

\section{Introduction}

Green tea, originated in China as a medicinal drink, is a globally popular beverage made from cured leaves of the Camellia sinensis. It was claimed in book named The Divine Farmer's Herb-Root Classic that Camellia sinensis infusions were helpful for treating many different kinds of disease conditions. Modern scientific research also revealed that green tea may be effective as an antioxidant, anti-inflammatory, anti-carcinogenic, and as an antimicrobial [1,2].

Tea leaves contain diverse polyphenols (i.e. catechins), saponins and some protein nutrients $[3,4,5]$. Of these compounds, polyphenols are well studied as the active ingredients with antioxidant and anti-inflammatory properties [6]. Anti-carcinogenic property of polyphenols, probably caused by their antioxidant and antiinflammatory effects, has also been found in many types of cancer [1]. The mechanisms may include inducing apoptosis in cancer cells and inhibiting angiogenesis [1], $[7,8]$.
Carcinogenesis is a complex and multistep process in which distinct molecular and cellular modifications arise. Among these cellular processes, angiogenesis plays important roles by providing tumor with essential nutrients and oxygen, and a route for metastasis $[9,10,11,12]$. Thus, anti-angiogenesis has been considered as one of the most important anticancer therapies. In comparison with chemotherapy directed at cancer cells, which often rapidly mutate and acquire "drug resistance" to treatment, the antiangiogenic therapy is obviously advantageous [13].

Compared with well-studied tea polyphenols, less literature data is available about the pharmacological activities of tea saponin (TS). So, we are interested if the anticancer effect of tea leaf is associated with its saponin and its effect on angiogenesis. In this present study, we first investigated the in vitro anti-angiogenic properties of TS and tea aglucone (TA), by analyzing their effects on the proliferation, migration, invasion, and tube formation of human umbilical vein endothelial cells (HUVECs). It was found that both TS and TA could inhibit angiogenesis relevant processes of HUVECs, even TA acted at higher doses with lower effect. 


\section{Methods}

\subsection{Chemicals}

TS and TA were obtained from Laboratory of Ethnopharmacology, Regenerative Medicine Research Center, West China Hospital/West China Medical School, Sichuan University. The purities of TS and TA are above 95\% by high-performance liquid chromatography analysis. Figure 1 shows the structure of TS and TA.

\subsection{Cell Culture}

HUVECs were isolated from human umbilical cord veins by a standard procedure, as previously described [14], and grown in EBM-2 medium with EGM-2 SingleQuots containing VEGF and other growth factors (Lonza, USA). HUVECs at passages 3 to 8 were used for all experiments.

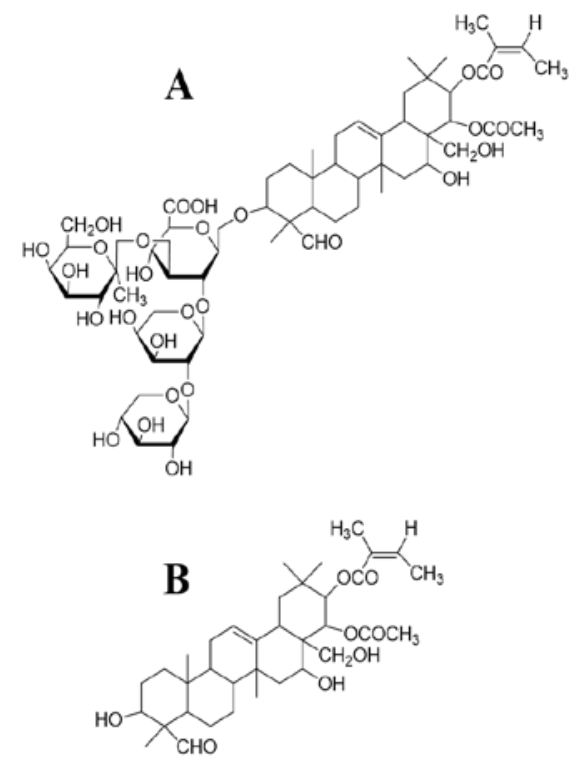

Figure 1. Chemical structures of TS (A) and TA (B)

\subsection{Cell Proliferation Assay}

Cell proliferation was analyzed using Cell Counting Assay Kit-8 (CCK-8) (Dojindo Molecular Technologies, Japan) according to the manufacturer's protocol. Briefly, $100 \mu 1$ of cell suspension $\left(5 \times 10^{3}\right.$ cells/well $)$ was dispensed into a 96-well plate and pre-incubated overnight, then exposed to various concentrations of TS or TA for $24 \mathrm{~h}$. Ten microliters of CCK-8 solution was added to each well. After incubation for $1 \mathrm{~h}$, the absorbance at $450 \mathrm{~nm}$ was measured using microplate reader. Experiments were performed at least three times with representative data presented. The inhibition percentage of TS or TA on cell proliferation was expressed using vehicle treated cells at $100 \%$.

\subsection{Cell Cycle Analysis}

Cell cycle analysis was carried out with sub-confluent HUVECs. After incubation with vehicle, various concentrations of TS for $24 \mathrm{~h}$, HUVECs were harvested, washed twice with cold PBS (pH 7.4), fixed with 70\% ethanol overnight, washed twice again and then stained with propidium iodide (PI) using a Cycle TEST PLUS
DNA Reagent Kit (BD Pharmingen, USA), finally subjected to flow cytometry with a FACScan flow cytometer (Becton Dickinson, USA). Thirty thousand events were collected for each sample. The number of gated cells in the G1, G2/M or S-phase is presented as percentage.

\subsection{Cell Apoptosis Detection}

Cell apoptosis analysis was performed using Annexin V/PI apopotosis assay kit (KeyGEN Biotech, China) according to the manufacturer's protocol. Briefly, after incubation with vehicle, various concentrations of TS for 24 h, HUVECs were harvested, washed twice with cold PBS (pH 7.4), and stained with $3 \mu$ of Annexin V and $3 \mu \mathrm{l}$ of PI in $300 \mu$ of binding buffer for $10 \mathrm{~min}$ in the dark. Cell apoptosis was then analyzed by Becton Dickinson FACScan Flow Cytometer (Becton Dickinson).

\subsection{Transmission Electron Microscopy (TEM)}

Cells were fixed in cold $\left(4^{\circ} \mathrm{C}\right) 3 \%$ phosphate-buffered glutaraldehyde $(\mathrm{pH}$ 7.2) for $2 \mathrm{~h}$, and post fixed for a further $2 \mathrm{~h}$ in $1 \%$ phosphate-buffered osmic acid. The sample was dehydrated in acetone and finally embedded in Epon812 (SPI). Ultrathin (50-70 nm) sections were cut and double-stained with uranyl acetate and lead citrate. Cells were examined on Hitachi transmission electron microscope system (Japan).

\subsection{Wound Healing Migration Assay}

HUVECs were allowed to grow to full confluence in 24-well plates, then wounded by scratching with pipette tips and washed with PBS. Serum-free EGM-2 medium containing vehicle, different concentrations of TS or TA were added to the scratched monolayers. Images were taken using an OLYMPUS digital camera after $16 \mathrm{~h}$ of cell migration. The migrated cells were quantified by manual counting, and the percentage of inhibition was expressed on the basis of vehicle treated cells.

\subsection{Transwell Invasion Assay}

Invasion assay was carried out as described previously [13]. In brief, the filter of the Transwell plate (Corning, USA) was coated with $50 \mu$ l Matrigel (BD Biosciences). After Matrigel polymerization, the bottom chambers were filled with EGM-2 medium containing various growth factors, and the top chambers containing $100 \mu \mathrm{l}$ EBM-2 medium (without growth factors) were seeded with HUVECs $\left(2 \times 10^{4}\right.$ cells/well $)$ with vehicle, various concentrations of TS or TA. After invading for $24 \mathrm{~h}$, noninvaded cells were scraped with a cotton swab, and invaded cells were fixed with methanol and stained with $0.05 \%$ crystal violet. The cells were photographed under a light microscope and quantified by manual counting. The inhibition percentage of TS or TA on cell invasion was expressed using vehicle treated cells at $100 \%$.

\subsection{Tube Formation Assay}

The tube formation assay was performed as described previously [15]. Assessment of in vitro capillary tube-like formation was carried out using a growth factor-reduced basement membrane Matrigel matrix (BD Biosciences). 
Fifty microliters of Matrigel were distributed as a thin layer onto the bottom of 48-well cell plates and left for polymerization at $37^{\circ} \mathrm{C}$ for 30 min. HUVECs suspended in EGM-2 medium were seeded onto the Matrigel in the presence of vehicle or different concentrations of TS or TA. After $6 \mathrm{~h}$, cells were photographed under a light microscope. Three microscopic fields were selected at random, and the length of tube-like structures per-field was measured using Image-pro Plus 6.0 System (Media Cybernetics, USA). The percentage of inhibition was expressed using vehicle treated cells at $100 \%$.

\subsection{Statistical Analysis}

Data were expressed as mean \pm standard deviation (SD). Data were analyzed with one-way ANOVA using SPSS 16.0. Differences were considered statistically significant when $P<0.05$.

\section{Results}

\subsection{TS and TA Inhibit HUVEC Proliferation}

Angiogenesis, contributing to tumor growth and metastasis, is characterized by endothelial cell proliferation, migration

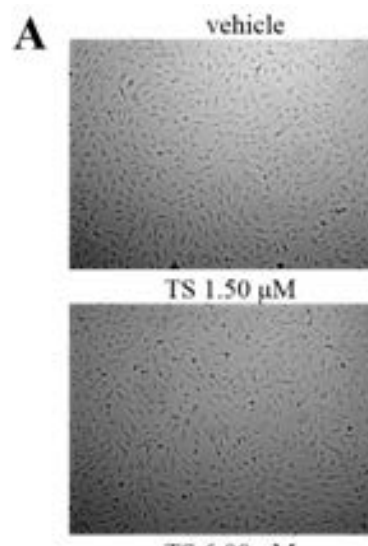

TS $6.00 \mu \mathrm{M}$

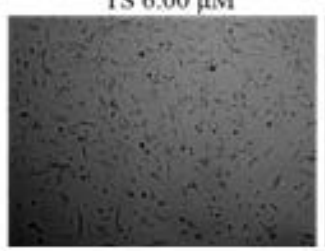

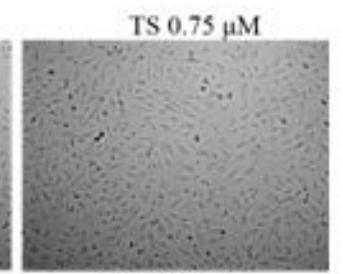

TS $3.00 \mu \mathrm{M}$

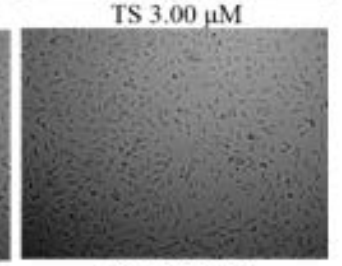

TS $12.00 \mu \mathrm{M}$

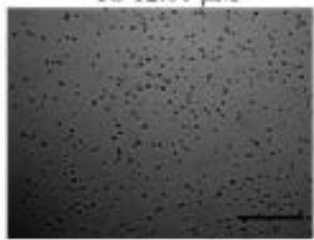

and tube formation [16,17]. The endothelial cell proliferation induced by vascular endothelial growth factor (VEGF) leads on to the angiogenesis process. To assess the antiangiogenic activity of TS and TA in vitro, their inhibitory effects on VEGF-induced HUVEC proliferation were first evaluated. The VEGF-induced HUVEC proliferation was obtained by treatment with $5 \mu \mathrm{g} / \mathrm{ml}$ VEGF-A.

It was found that treatment with TS induced morphologic changes in HUVECs at doses above $6 \mu \mathrm{M}$ for $24 \mathrm{~h}$ (Figure 2A). TA induced morphologic changes at doses above $25 \mu \mathrm{M}$ (data not presented). Cell viability was determined using CCK-8 assay. As shown in Figure 2B, treatment with TS resulted in the inhibition of VEGFinduced proliferation of HUVECs, with an IC50 value of $7.5 \pm 0.6 \mu \mathrm{M}$. TA inhibited VEGF-induced proliferation at a much higher concentration with an IC50 value of $25.3 \pm$ $1.2 \mu \mathrm{M}$ (Figure 2B).

The inhibitory effects of both TS and TA on VEGFinduced growth of HUVECs suggested their potential as anti-angiogenic agents. Considering the toxic effects of both TS and TA on the cell morphology of HUVECs, we investigated the inhibitory effects of TS and TA on the migration, invasion and tube formation of HUVECs using non-toxic doses below the values of their respective IC50.

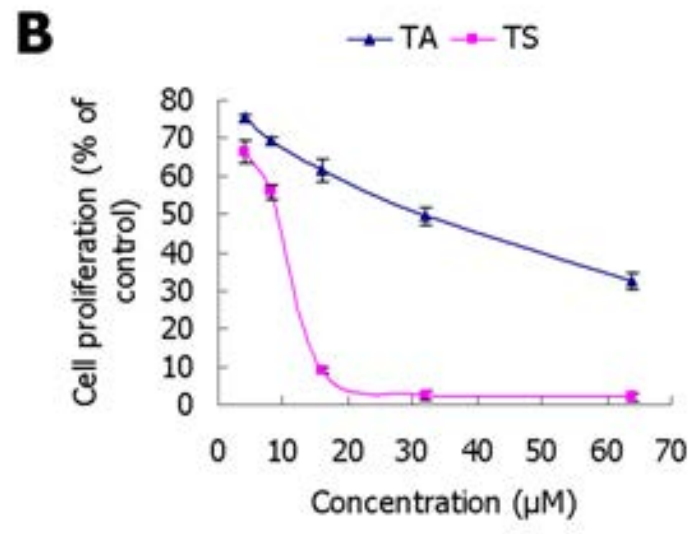

Figure 2. Effect of TS on HUVEC morphology (A) and VEGF-induced proliferation (B). (A) After treated with varying concentrations of TS for $24 \mathrm{~h}$, cell morphology was observed under inverted light microscopy. (B) VEGF-induced HUVEC proliferation was obtained by treatment with $5 \mu \mathrm{g} / \mathrm{ml}$ VEGF-A. After treated with varying concentrations of TS or TA for $24 \mathrm{~h}$ with presence of $5 \mu \mathrm{g} / \mathrm{ml}$ VEGF-A, cell viability was quantified using CCK-8 kit. Scale bar, $10 \mu \mathrm{m}$

\subsection{Effect of TS on HUVEC Cell Cycle Progression}

To understand the anti-proliferation effect of TS, we examined the cellular responses associated with cell death under TS treatment. Owing to the fact that interference of cell cycle progression would definitely result in cell proliferation inhibition [18], cell cycle analysis was performed using PI staining and flow cytometry. HUVECs were treated with TS $(3,6$, or $12 \mu \mathrm{M})$ for $24 \mathrm{~h}$, further stained and examined. Significant modulation of HUVEC cell cycle progression by TS was not observed (Figure 3).

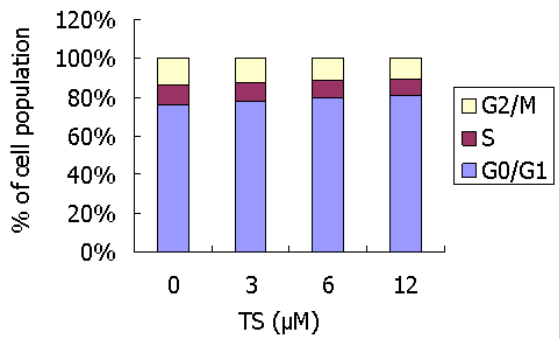

Figure 3. Graphical representation of cell cycle analysis of HUVECs. After treated with varying concentrations of TS for $24 \mathrm{~h}$, cell cycle analysis was performed using PI staining and flow cytometry. The cell population is expressed as percentage of the total cells analyzed. One representative experiment of three is shown 


\subsection{Effect of TS on HUVEC Apoptosis}

Cell death generally results from three different cellular processes: apoptosis, autophagy and necrosis [19]. To elucidate whether the growth-inhibitory effect of TS was related to the induction of apoptosis, flow cytometry analysis of Annexin V/PI stained HUVECs was performed. HUVECs were treated with TS (3, 6, or $12 \mu \mathrm{M})$ for $24 \mathrm{~h}$, further stained and examined. Representative histograms of HUVECs after treatment with TS were presented in Figure 4. It was found that there was no significant difference in the proportion of apoptotic cells between vehicle and TS treated HUVECs.

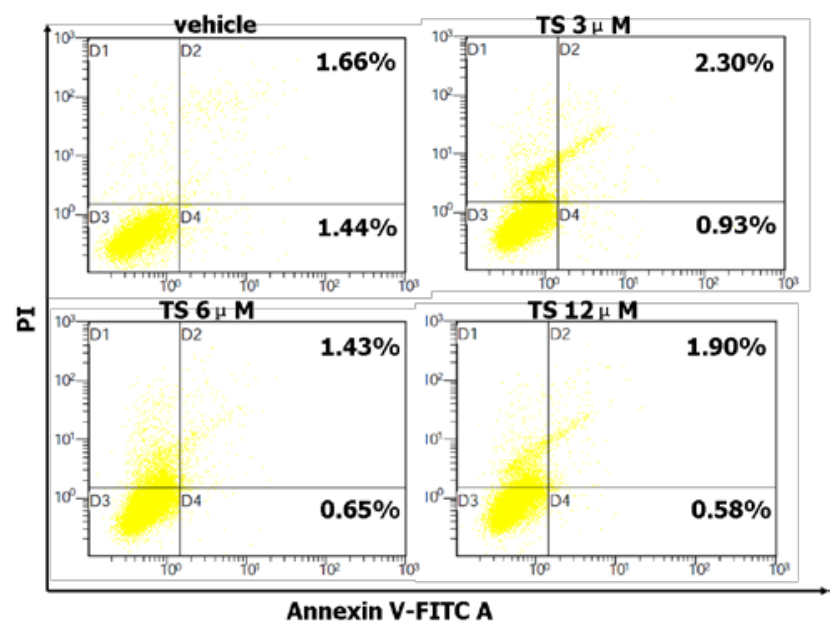

Figure 4. Flow cytometric evaluation of HUVEC apoptosis. Histograms derived from flow cytometry comparing apoptotic cells between vehicle and indicated concentrations of TS treated cells. After treatment for $24 \mathrm{~h}$, the induction of apoptosis was determined using flow cytometric analysis of Annexin V-FITC and PI-stained HUVECs. Cells in the lower right quadrant indicate Annexin-positive, early apoptotic cells, and cells in the upper right quadrant indicate Annexin-positive/PI positive, late apoptotic cells. Data were the mean result from three parallel experiments

\subsection{TS Induces Autophagic Vacuoles in HUVECS}

Like apoptosis, autophagy is also controlled tightly and plays a critical role in cell growth $[20,21]$. Owing to the fact that prolonged autophagy can lead to non-apoptotic programmed cell death, autophagy of HUVECs was hence investigated. HUVECs were treated with TS $(1.5,3$, or 6 $\mu \mathrm{M})$ for $24 \mathrm{~h}$ and further examined using TEM. Representative morphologies of HUVECs after treatment with $3 \mu \mathrm{M}$ of TS were presented in Figure 5. As shown in Figure 5, in contrast to the control group, numerous autophagic vacuoles were observed in HUVECs treated with TS. The vehicle treated control cells exhibited an undifferentiated phenotype, whereas in the TS-treated cells, various kinds of autophagic vesicles (white arrowheads in Figure 5B) were observed within the cytoplasm. It may be noted that there were varying autophagosomes, such as typical autophagosomes (Au in Figure 5C) with intracellular components and organelles, early autolysosomes (Figure 5D) with residual autophagosomes (Au) and lysosomes (Ly), and late autolysosomes (Figure 5E) with degraded amorphous substances.

It is worth mentioning that characteristics of apoptosis, such as cell shrinkage, nuclear condensation and fragmentation were not observed in TS treated HUVECs (Figure 5A and Figure 5B). The result of morphological observation is consistent with that of the apoptotic analysis with Annexin V/PI staining. Both results revealed no apoptotic induction by TS.

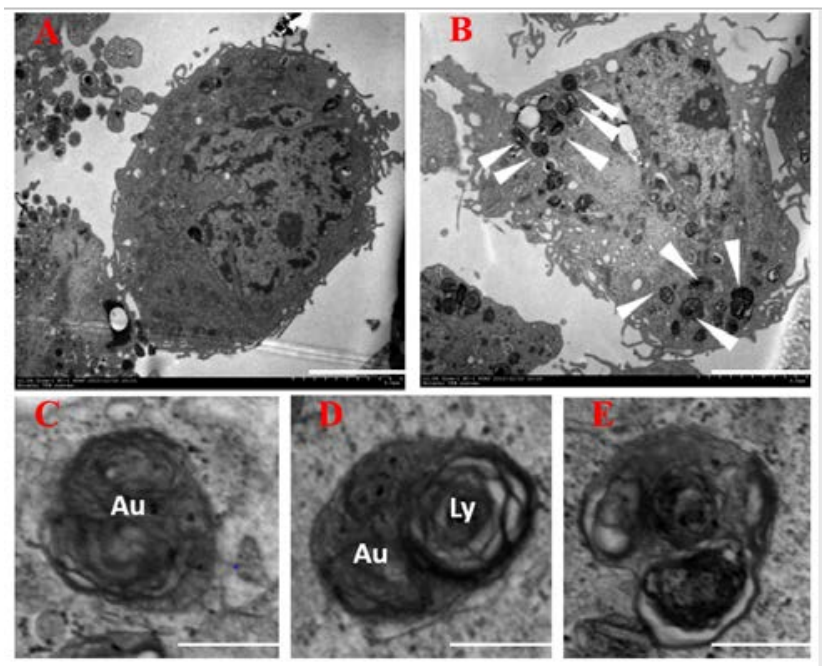

Figure 5. Transmission electron micrographs of HUVECs treated with vehicle (A) or $3 \mu \mathrm{M}$ (B-E) of TS for $24 \mathrm{~h}$. Control cells treated with vehicle exhibited a normal undifferentiated phenotype, showing absence of apoptotic bodies and nuclear condensation, whereas in the TS-treated cells, various kinds of autophagic vesicles (white arrowheads in B) are observed within the cytoplasm. These vesicles include the typical autophagosomes ( $\mathrm{Au}$ in $\mathrm{C}$ ), early autolysosomes (D) in which lysosomes (Ly) are still present, and late autolysosomes (E) with degraded amorphous substances. Scale bars represent $5 \mu \mathrm{m}$ in A and B and $0.5 \mu \mathrm{m}$ in $\mathrm{C}-\mathrm{E}$

\subsection{TS and TA Inhibit HUVEC Migration}

Endothelial cell migration is a key step for tube formation in tumor angiogenesis and tumor metastasis $[16,17]$. In this work, wound healing assays were carried out to investigate the effects of TS on HUVEC migration. The result revealed that TS inhibited the migration of HUVECs in a dose-dependent manner (Figure 6A). Treatment with TS at 1.5 and $3 \mu \mathrm{M}$ reduced cell migration by $28.2 \%$ and $46.7 \%$, respectively (Figure 6A). The results indicated that TS significantly $(P<0.001)$ inhibited the migration of HUVECs.

For comparison, the inhibition effect of TA on HUVEC migration was also investigated. The result revealed that TA also inhibited the migration of HUVECs in a dosedependent manner (Figure 6B). Treatment with 7.5 and 15 $\mu \mathrm{M}$ of $\mathrm{TA}$ reduced cell migration by $8.3 \%$ and $21.9 \%$, respectively (Figure 6B). Compared with TS, TA acted at higher doses with much lower effect on HUVEC migration.

\subsection{TS and TA Inhibit HUVEC Invasion}

The invasion of HUVECs across the extracellular matrix is also a fundamental step during angiogenesis $[16,17]$. To examine the inhibitory effect of TS on the invasion of HUVECs, Transwell invasion assays were conducted to evaluate the ability of HUVECs, treated with varying concentrations of TS, on passing through the Matrigel and membrane barrier of the Transwell. The result showed that TS inhibited the invasion property of HUVECs in a dose-dependent manner (Figure 7A). Treatment with TS at 1.5 and $3 \mu \mathrm{M}$ resulted in inhibition of cell invasion by $31.0 \%$ and $72.6 \%$ respectively (Figure 7A). 
The results demonstrated that TS significantly inhibited migration of HUVECs $(P<0.001)$.

For comparison, the inhibitory effect of TA on HUVEC invasion was also investigated. The result revealed that TA also inhibited the invasion of HUVECs in a dosedependent manner (Figure 7B). Treatment with TA at 7.5 and $15 \mu \mathrm{M}$ reduced cell invasion by $10.3 \%$ and $27.1 \%$, respectively (Figure 7B). Compared with TS, TA acted at higher doses with lower effect on HUVEC invasion.

A
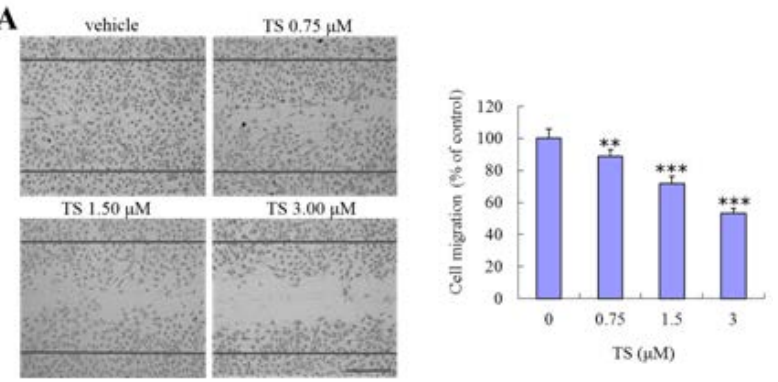

B
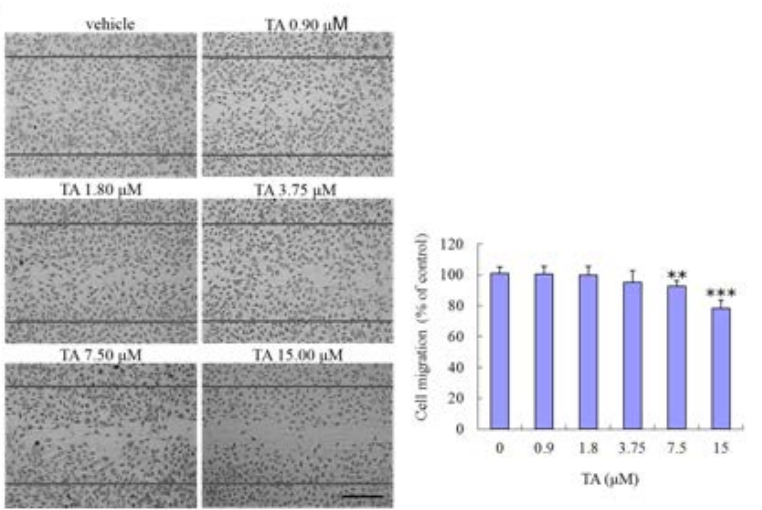

Figure 6. Effect of TS (A) and TA (B) on HUVEC migration in wound healing assay. Monolayer HUVECs were wounded by scratching with pipette tips and treated with ethanol vehicle or different concentrations of TS or TA in serum-free EGM-2 medium. After $16 \mathrm{~h}$ of incubation, the migrated cells were quantified by manual counting. The percentage of inhibition was expressed using vehicle treated cells at $100 \%$. The results are expressed as mean $\pm S D(n=5)$. Columns, mean; bars, $S D$. **, $P<0.01$; $* * *, P<0.001$ versus vehicle-treated group. Scale bar, $10 \mu \mathrm{m}$

\subsection{TS and TA Inhibit HUVEC Tube Formation}

Although angiogenesis is a complex and multistep process with several kinds of cells involved, tube formation of endothelial cells is one of the crucial steps $[16,17]$. To investigate the inhibitory effect of TS on angiogenesis, we carried out in vitro tube formation assay of HUVECs on the surface of Matrigel. The results revealed that HUVECs formed capillary-like structures on the surface of Matrigel in the control group within $6 \mathrm{~h}$. Treatment with TS dose-responsively inhibited the tube formation (Figure 8A). Treatment with TS at 1.5 and 3 $\mu \mathrm{M}$ resulted in tube length reduction by $54.1 \%$ and $65.3 \%$ respectively (Figure 8A). The results demonstrated that TS significantly inhibited tube formation property of HUVECs $(P<0.001)$.

For comparison, the inhibitory effect of TA on HUVEC tube formation was also investigated. The result revealed that TA also inhibited the tube formation of HUVECs in a dose-dependent manner (Figure 8B). Treatment with TA at 7.5 and $15 \mu \mathrm{M}$ reduced tube length by $16.0 \%$ and $27.5 \%$, respectively (Figure 8B). Compared with TS, TA acted at higher doses with lower effect on HUVEC tube formation property.

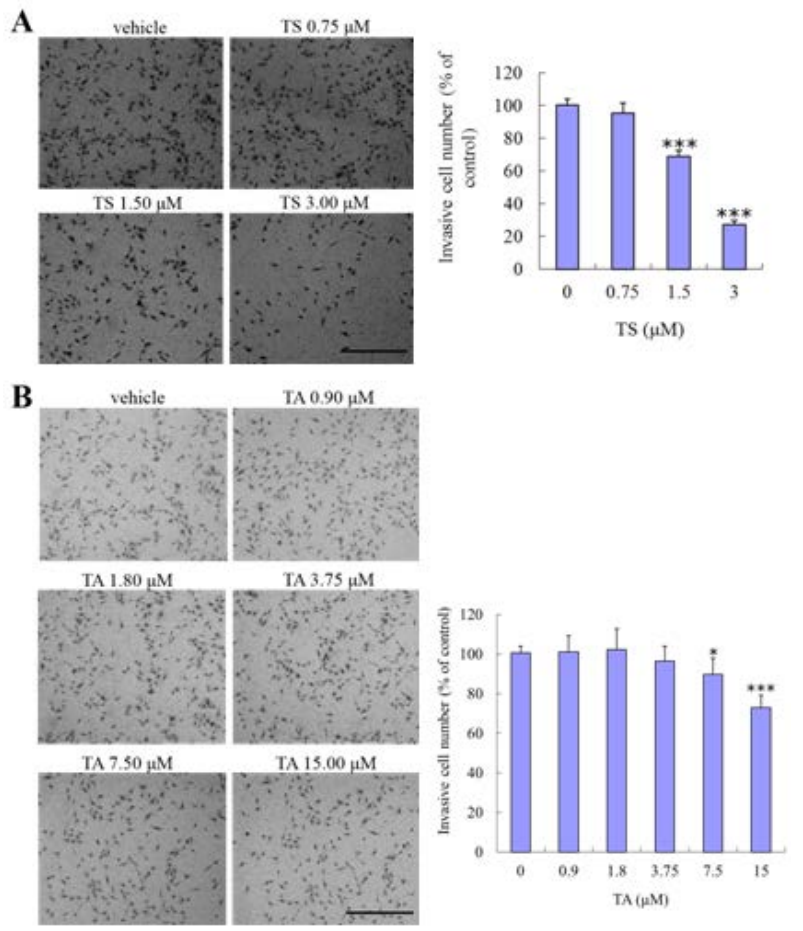

Figure 7. Effect of TS (A) and TA (B) on HUVEC invasion in Transwell assay. A total of $2 \times 10^{4}$ HUVECs were seeded in the top chamber and treated with ethanol vehicle or varying concentrations of TS or TA. After $24 \mathrm{~h}$, HUVECs that invaded through the membrane were stained with crystal violet and quantified. The percentage of inhibition was expressed using vehicle treated cells at $100 \%$. The results are expressed as mean \pm $S D(n=5)$. Columns, mean; bars, $S D$. *, $P<0.05$; ***, $P<0.001$ versus vehicle-treated group. Scale bar, $10 \mu \mathrm{m}$

\section{Discussion}

Green tea is an extremely popular drink in the world. Its habitual consumption has long been associated with health benefits including antioxidant, anti-inflammatory and anticarcinogenic efficacy [1]. The effects of green tea on cancer, mostly chemo-preventive effects, are generally thought to be mediated by its polyphenols known as catechins [22,23]. Compared with the well-studied tea polyphenols, less attention was paid to the health effects of TS, which is an important component of Camellia sinensis.

In green tea leaves, several saponins have already been identified, including theasaponin B1 [24], assamsaponin J [25], isotheasaponin B1-B3 [26], foliatheasaponin I-IV and floratheasaponin A [27]. Pharmacological studies have revealed that tea-leaf saponin might have antimicrobial [28], anti-inflammatory [28], anti-allergic [29] and antihypercholesterolemic effects [30]. In this work, we evaluated the in vitro anti-angiogenic effect of TS and TA for the first time. Our data indicated the concentrationdependently inhibitory effects of TS and TA on HUVEC proliferation, migration, invasion and tube formation.

For endothelial cells that undergo angiogenesis, the involved cellular functions are defined as proliferation, migration and invasion [16,17]. The well-defined mechanisms of existing anti-angiogenic agents would involve the interferences of those cellular processes of 
HUVECs [31,32]. For angiogenesis inhibitors, interference of VEGF-induced HUVECs proliferation is a common phenomenon [31]. In this work, both TS and TA inhibited the VEGF-induced growth of HUVECs in a dose-responsive manner (Figure 2). Notwithstanding the drug induced morphologic changes of HUVECs revealed the cell toxic effects of both TS and TA, their inhibitory effects on VEGF-induced growth of HUVECs suggested their potential as antiangiogenic agents.

To avoid the toxic effects, the inhibitory effects of TS and TA on migration, invasion and tube formation of HUVECs were investigated using non-toxic doses below the values of their respective IC50. Notably, the inhibition by TS and TA occurs at concentrations below those that show significant direct effects on the normal growth of endothelial cell. One and a half or $3 \mu \mathrm{M}$ of TS were sufficient to obviously block migration, invasion and capillary-like structure formation in vitro. Seven and a half or $15 \mu \mathrm{M}$ of TA also resulted in the inhibition of migration, invasion and tube formation of HUVECs with relatively lower effect. The inhibitory properties of TS and TA on these cellular processes have shown promise of anti-angiogenic potency for tea leaf.
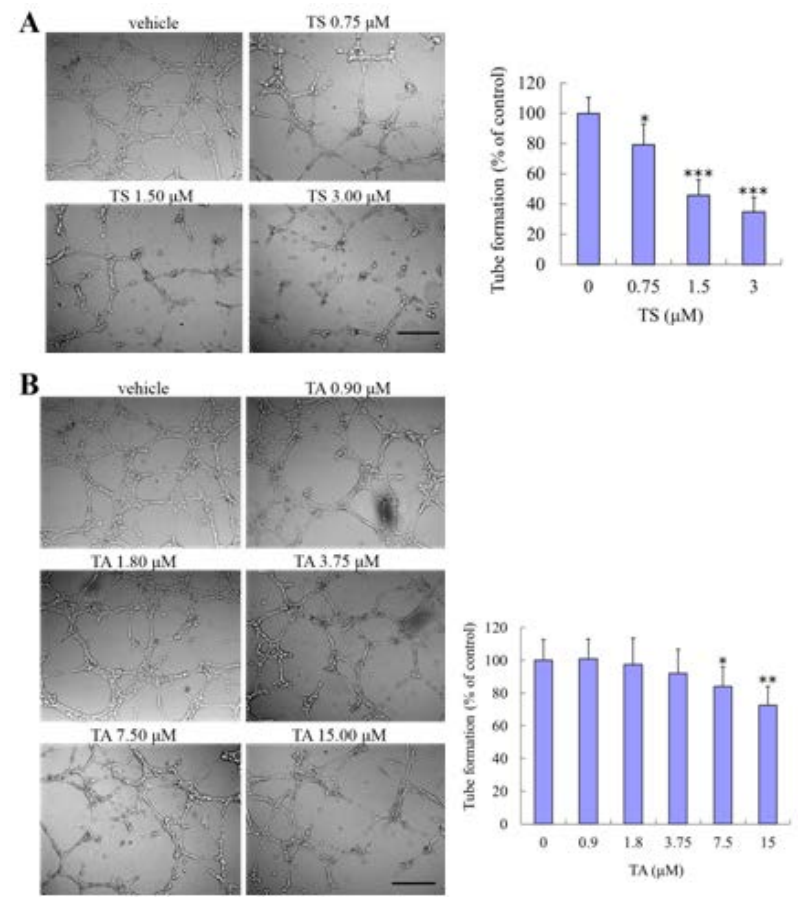

Figure 8. Effect of TS (A) and TA (B) on HUVEC tube formation. After treated with vehicle or varying concentrations of TS or TA for $6 \mathrm{~h}$, tubular structure in each group was measured using Image-pro Plus 6.0 System. The percentage of inhibition was expressed using vehicle treated cells at $100 \%$. The results are expressed as mean $\pm S D(n=3)$. Columns, mean; bars, $S D$. *, $P<0.05$; **, $P<0.01$, ***, $P<0.001$ versus vehicletreated group. Scale bar, $10 \mu \mathrm{m}$

The differences in anti-angiogenic potencies between TS and TA might result from their bioavailability variances. Owing to the well-known fact that low availability of a medication is a great barrier to its efficacy, in order for any of the components of green tea to be a health benefit they have to have bioavailability. Compared with TA, the relatively higher efficiency of TS at low dosage might be attributed to improvements in solubility and tight junction opening [33], or higher ability of membrane transportation owing to the presence of glycosyl moieties [34].
Cell death and growth inhibition generally results from three different cellular processes: apoptosis, autophagy and necrosis [18]. In addition, interference of cell cycle progression would definitely result in cell proliferation inhibition. Presently, many kinds of steroidal saponinshave been reported to induce apoptosis in a wide variety of tumor cells through cell cycle arrest [35], activation of p53 and caspase-3 [36,37], etc. Here, TS induced no apoptosis, but obvious autophagic process in HUVECs, with no effect on its cell cycle. When TStreated HUVECs were investigated under a transmission electron microscope, characteristic autophagosomes and autolysosomes were present (Figure 5.B-E).

Like apoptosis, autophagy is also controlled tightly and plays a critical role in tissue homeostasis, development and disease [20,21]. It is a cellular defense process that involves the sequestration and delivery of cytoplasmic material by autophagosomes to the lysosome where it is degraded and recycled $[38,39]$. Although autophagy is initiated as a protective response to stress, persistent autophagy can lead to growth inhibition and cell death. The autophagic vacuoles in the TS-treated HUVECs might suggest the involvement of autophagic induction in the growth inhibition of HUVECs.

Recently, VEGF receptor 2 (VEGFR2) - mediated antiangiogenic effects of two steroid saponins, Theasaponin E1 (present in tea seed) and deltonin, have been reported separately [40,41]. Consistent with deltonin and Theasaponin E1, TS inhibited the cellular processes of angiogenesis in vitro, including HUVEC migration, invasion, and tube formation. Its underlying mechanism might also be related with suppression of VEGF/VEGFR2 signal pathway. It's interesting that we found TS inhibited angiogenesis related processes at the same lower dose (3 $\mu \mathrm{M})$ as that induced autophagy, suggesting that the antiangiogenic effects of TS might be related with its ability to induce autophagy. The growth inhibition of HUVECs induced by persistent autophagy would further induce the inhibition of HUVEC migration, invasion, and tube formation. Taken together, our results suggest that TS may have multi-target action as an anti-angiogenic agent.

All together, the findings of this study indicated that TS and TA, derived from green tea, were effective in inhibiting HUVEC proliferation, migration, invasion and tube formation. The mechanism might be associated with the induction of autophagic process. In future work, the utilization of autophagy inhibitors would help us investigate the mechanism by which TS inhibits angiogenesis either through directly modulating angiogenic process, or activating autophagy which would then lead to inhibited angiogenesis. Our data about the anti-angiogenic effects of TS and TA, presented for the first time, would provide a new insight into the health potential for green tea.

\section{Statement of Conflicts of Interest}

The authors disclose no potential conflicts of interest.

\section{Acknowledgement}

The present study was supported by the China National '12.5' Foundation (no. 2011BAJ07B04) and the National Natural Science Foundation of China (no. 20972105). 


\section{References}

[1] Serafini, M., DelRio, D., Yao, D.N., Bettuzzi, S., and Peluso, I., "Health benefits of tea", in Herbal Medicine: Biomolecular and Clinical Aspects, $2^{\text {nd }}$ Edn, Chapter 12, eds Benzie, I.F.F., and Wachtel-Galor, S., 2011.

[2] Reygaert, W.C., "The antimicrobial possibilities of green tea," Front Microbiol, 5. 434. Aug.2014.

[3] Ferruzzi, M.G., "The influence of beverage composition on delivery of phenolic compounds from coffee and tea," Physiol Behav, 100 (1). 33-41. Apr.2010.

[4] Williamson, G., Dionisi, F., and Renouf, M., "Flavanols from green tea and phenolic acids from coffee: critical quantitative evaluation of the pharmacokinetic data in humans after consumption of single doses of beverages," Mol Nutr Food Res, 55(6). 864-873. Jun.2011.

[5] Matsui, Y., Kobayashi, K., Masuda, H., Kigoshi, H., Akao, M., Sakurai, H., and Kumagai, H., "Quantitative analysis of saponins in a tea-leaf extract and their antihypercholesterolemic activity," Biosci Biotechnol Biochem, 73(7). 1513-1519. Jul.2009.

[6] Katiyar, S.K., Afaq, F., Perez, A., and Mukhtar, H., "Green tea polyphenol (-)-epigallocatechin-3-gallate treatment of human skin inhibits ultraviolet radiation-induced oxidative stress," Carcinogenesis, 22(2). 287-294. Feb.2001.

[7] Jigisha, A., Nishant, R., Navin, K., and Pankaj, G., "Green tea: a magical herb with miraculous outcomes," Int Res J Pharm, 3. 139148. 2012.

[8] Subramani, C., and Natesh, R.K., "Molecular mechanisms and biological implications of green tea polyphenol, (-)-epigallo catechin-3-gallate," Int J Pharm Biosci Tech, 1. 54-63. 2013.

[9] Folkman, J., "Angiogenesis in cancer, vascular, rheumatoid and other disease,” Nat Med, 1(1). 27-31. Jan.1995.

[10] Carmeliet, P., and Jain, R.K., "Angiogenesis in cancer and other diseases,” Nature, 407(6801).249-257. Sep.2000.

[11] Bergers, G., and Benjamin, L.E., "Tumorigenesis and the angiogenic switch," Nat Rev Cancer, 3(6).401-410. Jun.2003.

[12] Folkman, J., "Role of angiogenesis in tumor growth and metastasis,” Semin Oncol, 29 (6 Suppl 16).15-18. Dec.2002.

[13] Zhang, S., Cao, Z., Tian, H., Shen, G., Ma, Y., Xie, H., Liu, Y., Zhao, C., Deng, S., Yang, Y., Zheng, R., Li, W., Zhang, N., Liu, S., Wang, W., Dai, L., Shi, S., Cheng, L., Pan, Y., Feng, S., Zhao, X., Deng, H., Yang, S., and Wei, Y., "SKLB1002, a novel potent inhibitor of VEGF receptor 2 signaling, inhibits angiogenesis and tumor growth in vivo," Clin Cancer Res, 17(13). 4439-4450. Jul.2011.

[14] Jaffe, E.A., Nachman, R.L., Becker, C.G., and Minick, C.R., "Culture of human endothelial cells derived from umbilical veins. Identification by morphologic and immunologic criteria," J Clin Invest, 52(11). 2745-2756. Nov.1973.

[15] Pang, X., Yi, Z., Zhang, X., Sung, B., Qu, W., Lian, X., Aggarwal, B.B., and Liu, M., "Acetyl-11-ketob-boswellic acid inhibits prostate tumor growth by suppressing vascular endothelial growth factor receptor 2-mediated angiogenesis,” Cancer Res, 69(14). 5893-5900. Jul.2009.

[16] Polverini, P.J., "The pathophysiology of angiogenesis," Crit Rev Oral Biol Med, 6(3). 230-247.1995.

[17] Bischoff, J., "Cell adhesion and angiogenesis," J Clin Invest, 99(3). 373-376. Feb.1997.

[18] Kaufmann, W.K, and Kaufman, D.G., "Cell cycle control, DNA repair and initiation of carcinogenesis," FASEB J, 7(12). 11881189. Sep.1993.

[19] Kitanaka, C., and Kuchino, Y., "Caspase-independent programmed cell death with necrotic morphology," Cell Death Differ, 6(6). 508-515. Jun.1999.

[20] Kondo, Y., Kanzawa, T., Sawaya, R., and Kondo, S., "The role of autophagy in cancer development and response to therapy," Nat Rev Cancer, 5(9). 726-734. Sep.2005.

[21] Lum, J.J., DeBerardinis, R.J., and Thompson, C.B., "Autophagy in metazoans: cell survival in the land of plenty,” Nat Rev Mol Cell Biol, 6(6). 439-448. Jun.2005.

[22] Siddiqui, I.A., Asim, M., Hafeez, B.B., Adhami, V.M., Tarapore, R.S., and Mukhtar, H., "Green tea polyphenol EGCG blunts androgen receptor function in prostate cancer," FASEB J, 25(4). 1198-1207. Apr. 2011.

[23] Bettuzzi, S., Brausi, M., Rizzi, F., Castagnetti, G., Peracchia, G., and Corti, A., "Chemoprevention of human prostate cancer by oral administration of green tea catechins in volunteers with high-grade prostate intraepithelial neoplasia: a preliminary report from a one- year proof-of-principle study,” Cancer Res, 66(2).1234-1240. Jan.2006.

[24] Sagesaka, Y.M., Uemura, T., Watanabe, N., Sakata, K., and Uzawa, J., "A new glucuronide saponin from tea leaves (Camellia sinensis var. sinensis)," Biosci Biotechnol Biochem, 58(11). 2036-2040. Nov.1994.

[25] Murakami, T., Nakamura, J., Kageura, T., Matsuda, H., and Yoshikawa, M., "Bioactive saponins and glycosides. XVII. Inhibitory effect on gastric emptying and accelerating effect on gastrointestinal transit of tea saponins: structures of assamsaponins F, G, H, I, and J from the seeds and leaves of the tea plant," Chem Pharm Bull, 48(11).1720-1725. Nov.2000.

[26] Kobayashi, K., Teruya, T., Suenaga, K., Matsui, Y., Masuda, H., and Kigoshi, H., "Isotheasaponins B1-B3 from Camellia sinensis var. sinensis tea leaves,” Phytochemistry, 67(13). 1385-1389. Jul.2006.

[27] Morikawa, T., Nakamura, S., Kato, Y., Muraoka, O., Matsuda, H., and Yoshikawa, M., "Bioactive Saponins and Glycosides. XXVIII. New Triterpene Saponins, Foliatheasaponins I, II, III, IV, and V, from Tencha (the Leaves of Camellia sinensis)," Chem Pharm Bull, 55(2). 293-298. Feb.2007.

[28] Sagesaka, Y.M., Uemura, T., Suzuki, Y., Sugiura, T., Yoshida, M., Yamaguchi, K., and Kyuki, K., "Antimicrobial and antiinflammatory actions of tea-leaf saponin,"Yakugaku Zasshi (in Japanese), 116(3). 238-243. Mar.1996.

[29] Akagi, M., Fukuishi, N., Kan, T., Sagesaka, Y.M., and Akagi, R., "Anti-allergic effect of tea-leaf saponin (TLS) from tea leaves (Camellia sinensis var. sinensis)," Biol Pharm Bull, 20(5), 565567. May.1997.

[30] Matsui, Y., Kobayashi, K., Masuda, H., Kigoshi, H., Akao, M., Sakurai, H., and Kumagai, H., "Quantitative analysis of saponins in a tea-leaf extract and their antihypercholesterolemic activity," Biosci Biotechnol Biochem, 73(7). 1513-1519. Jul.2009.

[31] Xia, Y., Song, X., Li, D., Ye, T., Xu, Y., Lin, H., Meng, N., Li, G., Deng, S., Zhang, S., Liu, L., Zhu, Y., Zeng, J., Lei, Q., Pan, Y., Wei, Y., Zhao, Y., and Yu, L., "YLT192, a novel, orally active bioavailable inhibitor of VEGFR2 signaling with potent antiangiogenic activity and antitumor efficacy in preclinical models," Sci Rep, 4. 6031. Aug.2014.

[32] Muslim, N.S., Nassar, Z.D., Aisha, A.F., Shafaei, A., Idris, N., Majid, A.M., and Ismail, Z., "Antiangiogenesis and antioxidant activity of ethanol extracts of Pithecellobium jiringa," $B M C$ Complement Altern Med, 12. 210. Nov.2012.

[33] Okawara, M., Tokudome, Y., Todo, H., Sugibayashi, K., and Hashimoto, F., "Effect of $\beta$-cyclodextrin derivatives on the diosgenin absorption in Caco-2 cell monolayer and rats," Biol Pharm Bull, 37(1).54-59. 2014.

[34] Okawara, M., Tokudome, Y., Todo, H., Sugibayashi, K., and Hashimoto, F., "Enhancement of diosgenin distribution in the skin by cyclodextrin complexation following oral administration," Biol Pharm Bull, 36(1). 36-40. 2013.

[35] Liu, M.J., Wang, Z., Ju, Y., Wong, R.N., and Wu, Q.Y., "DiosTA induces cell cycle arrest and apoptosis in human leukemia K562 cells with the disruption of $\mathrm{Ca}^{2+}$ homeostasis," Cancer Chemother Pharmacol, 55(1). 79-90. Jan.2005.

[36] Raju, J., Patlolla, J.M., Swamy, M.V., and Rao, C.V., "Diosgenin, a steroid saponin of Trigonella foenum graecum (Fenugreek), inhibits azoxymethane-induced aberrant crypt foci formation in F344 rats and induces apoptosis in HT-29 human colon cancer cells," Cancer Epidemiol Biomarkers Prev, 13(8).1392-1398. Aug.2004.

[37] Leger, D.Y., Liagre, B., Cardot, P.J., Beneytout, J.L., and Battu, S., "DiosTA dosedependent apoptosis and differentiation induction in human erythroleukemia cell line and sedimentation field-flow fractionation monitoring,” Anal Biochem, 335(2). 267-278. Dec.2004.

[38] Yang, Z., and Klionsky, D.J., "An overview of the molecular mechanism of autophagy,” Curr Top Microbiol Immunol, 335.132. 2009.

[39] Mizushima, N., "Autophagy: process and function," Genes Dev, 21(22). 2861-2873. Nov.2007.

[40] Tong, Q., Zhao, Q., Qing, Y., Hu, X., Jiang, L., Wu, X., “Deltonin inhibits angiogenesis by regulating VEGFR2 and subsequent signaling pathways in endothelial cells," Steroids, 96C. 30-36. Dec.2014.

[41] Kim, J.D., Chaudhary, N., Seo, H.J., Kim, M.Y., Shin, T.S, "Theasaponin $\mathrm{E}_{1}$ as an effective ingredient for anti-angiogenesis and anti-obesity effects,” Biosci Biotechnol Biochem, 78(2). 279287. 2014. 